\title{
Author Correction: Protecting the ischaemic penumbra as an adjunct to thrombectomy for acute stroke
}

\section{Jean-Claude Baron}

Nature Reviews Neurology (2018) https://doi.org/10.1038/s41582-018-0002-2

Published online 19 April 2018

The originally published article contained an error in Table 1, whereby the RESCUE trial appeared on the "Transient descending aortic balloon occlusion (TAO)" line instead of the "Remote ischaemic perconditioning (RIPerC)" line. This has been corrected in the HTML and PDF versions of the manuscript.

https://doi.org/10.1038/s41582-019-0148-6 I Published online 11 February 2019 